\title{
Epidemiology, vaccine effectiveness, and risk factors for mortality for pneumococcal disease among hospitalised adults in Singapore: a case-control study
}

Tyson Chan ${ }^{1,2+}$, Min Zhi Tay ${ }^{1,2+}$, Win Mar Kyaw ${ }^{1}$, Angela Chow ${ }^{1}$ and Hanley J. Ho ${ }^{1 *}$ D

\begin{abstract}
Background: Streptococcus pneumoniae infections can lead to severe morbidity and mortality, especially in patients with invasive pneumococcal disease (IPD). This study evaluated factors associated with pneumococcal disease, pneumococcal vaccine effectiveness, and risk factors for all-cause mortality in hospitalised adults with pneumococcal disease in Singapore.

Methods: Retrospective case-control study of patients tested for pneumococcal disease with streptococcal urinary antigen testing and at least one sterile site culture, during their admission to a tertiary hospital in Singapore from 2015 to 2017. Patients were defined as cases of IPD or non-IPD, or as controls, based on laboratory results and clinical diagnoses. Multivariable models were constructed to determine factors associated with IPD/non-IPD, and risk factors for mortality from pneumococcal disease. Vaccine effectiveness against IPD/non-IPD was estimated using a variation of the test-negative design.
\end{abstract}

Results: We identified 496 pneumococcal disease cases, of whom 92 (18.5\%) had IPD. The mean age of cases was $69.1 \pm 15.4$ years, and $65.5 \%$ were male. Compared with controls $(N=9181)$, IPD patients were younger (mean age $61.5 \pm 16.3$ years, vs $72.2 \pm 16.1$ years in controls; $p<0.001$ ) and with less co-morbidities [median Charlson's score 1 (IQR 0-4), vs $3(1-5)$ in controls; $p<0.001]$. IPD patients also had the highest proportions with intensive care unit (ICU) admission (20.7\%), inpatient mortality (26.1\%) and longest median length of stay [9 (IQR 8-17) days]. On multivariable analysis, IPD was negatively associated with prior pneumococcal vaccination (adjusted relative risk ratio $=0.20,95 \% \mathrm{Cl} 0.06-0.69 ; p=0.011$ ). Risk factors for mortality among pneumococcal disease patients were ICU admission, diagnosis of IPD, age $\geq 85$ years and Charlson's score $>3$.

Conclusion: Patients with pneumococcal disease (especially IPD) were younger and had less co-morbidities than controls, but had higher risk of severe clinical outcomes and mortality. Pneumococcal vaccination effectiveness against IPD was estimated to be about $80 \%$, and should be encouraged among high-risk patients.

Keywords: Pneumococcal infections, Pneumococcal vaccines, Epidemiology, Mortality, Singapore

\footnotetext{
* Correspondence: hanleyho@gmail.com

${ }^{\dagger}$ Tyson Chan and Min Zhi Tay contributed equally to this work.

'Department of Clinical Epidemiology, Office of Clinical Epidemiology,

Analytics, and Knowledge, Tan Tock Seng Hospital, 11 Jalan Tan Tock Seng,

Singapore 308433, Singapore

Full list of author information is available at the end of the article
}

(c) The Author(s). 2020 Open Access This article is licensed under a Creative Commons Attribution 4.0 International License, which permits use, sharing, adaptation, distribution and reproduction in any medium or format, as long as you give appropriate credit to the original author(s) and the source, provide a link to the Creative Commons licence, and indicate if changes were made. The images or other third party material in this article are included in the article's Creative Commons licence, unless indicated otherwise in a credit line to the material. If material is not included in the article's Creative Commons licence and your intended use is not permitted by statutory regulation or exceeds the permitted use, you will need to obtain permission directly from the copyright holder. To view a copy of this licence, visit http://creativecommons.org/licenses/by/4.0/. The Creative Commons Public Domain Dedication waiver (http://creativecommons.org/publicdomain/zero/1.0/) applies to the data made available in this article, unless otherwise stated in a credit line to the data. 


\section{Background}

Pneumococcal disease is caused by Streptococcus pneumoniae and can lead to severe clinical outcomes and death. S. pneumoniae is the most common cause of community-acquired pneumonia (CAP), estimated to cause $27.3 \%$ of CAPs worldwide [1], and accounting for $29.2 \%$ of isolated pathogens from CAPs in Asia [2]. Other common sites of infection include otitis media and sinusitis. Invasive pneumococcal disease (IPD), defined as isolation of $S$. pneumoniae from a normally sterile site (such as blood or cerebrospinal fluid), carries an even higher risk of mortality and morbidity [3].

Cases of pneumococcal disease were predominantly male, and patients 65 years and above had the highest hospitalisation rates and case-fatality rates [4, 5]. From 1995 to 2004, the overall mean annual hospitalisation rate for pneumococcal disease in Singapore was 10.9 per 100,000 population and the case-fatality rate was $3.2 \%$ [4]. Specifically for IPD, locally reported case-fatality rates have ranged from 13.1 to $21.4 \%$ [6]

Transmission of the pneumococcus occurs via respiratory droplets from people with pneumococcal disease or asymptomatic individuals with carriage of the organism in their nasopharynx [7]. Risk factors for pneumococcal disease include presence of chronic diseases involving cardiovascular, pulmonary, hepatic, neurological or endocrine systems; immunosuppressed states; cigarette smoking history; alcohol abuse; recent influenza infection; institutionalised status; male sex and extremes of age [8]. Successful empiric antibiotic therapy is vital to avoid treatment failure and subsequent costs [9]. However, increasing antibiotic resistance in $S$. pneumoniae in the community has been reported [10]. Hence, while updated treatment recommendations (especially for $\mathrm{CAP}$ ) have reduced disease-associated complications and mortality, the risks from infection still remain significant [11].

Adult vaccination is an effective measure for upstream prevention of pneumococcal disease. Both the 13-valent pneumococcal conjugate vaccine (PCV13) (which covers for serotypes 1, 3, 4, 5, 6A, 6B, 7F, 9 V, 14, 18C, 19A, 19F and $23 \mathrm{~F}$ ) and 23-valent pneumococcal polysaccharide vaccine (PPSV23) (which covers for serotypes 1, 2, 3, 4, 5, 6B, 7F, 8, $9 \mathrm{~N}, 9 \mathrm{~V}, 10 \mathrm{~A}, 11 \mathrm{~A}, 12 \mathrm{~F}, 14,15 \mathrm{~B}, 17 \mathrm{~F}, 18 \mathrm{C}, 19 \mathrm{~A}, 19 \mathrm{~F}, 20$, 22F, 23F and 33F) [12], are effective against IPD [13-15]. PCV13 has also been shown to reduce the risk of subtypespecific non-invasive pneumococcal pneumonia [14]. Adult immunisation with both PCV13 and PPSV23 in elderly and high-risk groups is recommended by the United States Advisory Committee on Immunisation Practices (ACIP) [16]. The Singapore Ministry of Health (MOH) also recommended PPSV23 vaccination in adults in 2009 and subsequently both PCV13 and PPSV23 in 2017 [17]. However, pneumococcal vaccination uptake in adults has been low, estimated to be $6.1 \%$ among adults $\geq 65$ years in 2013 [18].
Previous studies in Asia have described the changing epidemiology of pneumococcal disease due to introduction of pneumococcal vaccines $[19,20]$. However, there have been few reports on the epidemiology of hospitalised adult patients with pneumococcal disease. Furthermore, evaluating the vaccine effectiveness in this group of patients may help strengthen the case for hospitalbased and nationwide adult vaccination programmes.

The aims of this study were to evaluate the factors associated with pneumococcal disease, vaccine effectiveness in preventing severe outcomes of pneumococcal disease, and the risk factors for all-cause mortality in hospitalised adults with pneumococcal disease in Singapore.

\section{Methods}

Patient population and study design

We conducted a case-control study on patients suspected to have pneumococcal disease admitted to Tan Tock Seng Hospital (TTSH), the second largest adult acute tertiary care hospital in Singapore with 1600 beds, from January 2015 through December 2017. Our participants comprised all patients (aged 16 years and above) admitted over the study period who were evaluated for pneumococcal disease using streptococcal urinary antigen testing and at least one sterile site clinical culture during their admission. Cases of pneumococcal disease were defined as either having i) a positive result on testing for streptococcal urinary antigen, or ii) growth of $S$. pneumoniae from sterile site cultures. For patients with multiple admissions for pneumococcal disease, only the first admission was included for this study.

We further stratified cases into those with IPD versus those with non-IPD. IPD was defined as either having i) a sterile site culture (e.g. blood, joint fluid, or pleural fluid) positive for $S$. pneumoniae, or ii) a positive streptococcal urinary antigen test and a clinical diagnosis suggesting invasive disease (e.g. meningitis, septic arthritis, or pleural empyema), with cultures from the relevant site negative for any other causative pathogen. A non-IPD case was defined as a patient with a positive streptococcal urinary antigen test, but without positive cultures or any clinical diagnosis suggesting invasive disease.

To identify factors associated with IPD and non-IPD, the cases were compared to a control group comprising the remainder of the participants. For clarity, controls were defined as patients admitted to TTSH over the same study period, with a negative test for streptococcal urinary antigen, and all laboratory cultures yielding negative results for S. pneumoniae. Again, for patients with multiple admissions, only the first admission fulfilling the definition was considered. Patients who did not have both streptococcal urinary antigen tests and at least one sterile site culture (typically, at least one blood 
culture) performed during their admission were excluded from this study.

\section{Data sources and classification}

All data for the study was captured using the hospital's electronic medical record system, which comprehensively captures the demographics, clinical characteristics, laboratory results, and vaccination records of patients. Pneumococcal vaccination was defined as ever having any record for PCV13 and/or PPSV23 given at least 14 days prior to the date of hospital admission. Inpatient mortality was defined as death resulting from any cause during the pneumococcal disease-related admission. International Classification of Diseases, 10th Revision (ICD-10) was used to establish patient diagnoses, comorbidities, risk factors, and infectious disease history. The co-morbidity status was assessed according to Charlson's co-morbidity score classification [21].

\section{Statistical analysis}

Descriptive statistics were analysed for all study participants and for patient subgroups, including the number and proportions for categorical characteristics, mean and standard deviation for normally distributed continuous variables, and median and interquartile range (IQR) for non-normally distributed variables.

We subsequently analysed differences in patient characteristics across our three patient subgroups (IPD, nonIPD and controls). Differences in proportions were assessed using Chi-square or Fisher's exact tests for categorical variables, while continuous variables were evaluated using the one-way ANOVA (for means) or KruskalWallis test (for medians) as appropriate. Multinomial regression analysis was used to further evaluate patient factors associated with IPD and non-IPD, as IPD, nonIPD and non-pneumococcal disease patients were considered as three unranked categories of patients, based on their laboratory results and clinical diagnosis. This also enabled us to analyse our entire study cohort using a single model. Factors to include in the model were determined based on a $p$ value threshold of 0.05 on univariate analysis, using non-pneumococcal disease patients as the base comparison group, with calculation of adjusted relative risk ratios (RRRs) for independent variables. Within our multivariable model, we included pneumococcal vaccination status (receipt of PCV13 and/ or PPSV23 vaccination at least 14 days prior to admission date) as a variable to determine its effectiveness in reducing risk of IPD / non-IPD. Vaccination effectiveness was calculated using the formula $[(1-R R R) \times$ $100 \%$ ], instead of the usual [(1-odds ratio, OR $) \times 100 \%$ ] used in the test-negative design. This was a variation of the test-negative design used for vaccine effectiveness studies, using multinomial logistic regression to construct the statistical model.

Among the cases, we also evaluated factors associated with inpatient all-cause mortality. Univariate analysis of demographic and clinical variables was first performed, and variables were included in a multiple logistic regression model again based on a $p$ value threshold of 0.05 on univariate analysis, with adjustment for demographic confounders. Unadjusted and adjusted odds ratios (ORs) and $95 \%$ confidence intervals (CIs) were calculated to determine the strength of association between variables and in-hospital all-cause mortality.

For all analyses, a $p$ value of $<0.05$ was considered statistically significant. Furthermore, inclusion of variables into multivariable logistic regression models also took into account confidence intervals and clinical significance of variables. All statistical analyses were performed using Stata version 13 (StataCorp 2013, College Station, TX).

\section{Results}

A total of 496 cases with pneumococcal disease were identified over the study period (Table 1). Cases were predominantly elderly (mean age $69.1 \pm 15.4$ years), male $(N=325,65.5 \%)$, and of Chinese ethnicity $(N=359$, 72.4\%). The median Charlson's comorbidity score was 2 (IQR 1-4). The most common co-morbidities were chronic pulmonary disease $(30.0 \%)$, diabetes mellitus (29.6\%), myocardial infarction/congestive cardiac failure $(28.8 \%)$, and renal disease (18.8\%). Majority had pneumonia as their primary diagnosis (70.0\%). Eighty (16.1\%) had received pneumococcal vaccination (PCV13 and/or PPSV23) at any time prior to admission. Ninety-two (18.5\%) cases had IPD. Within this group, 84 (91.3\%) had growth of $S$. pneumoniae in blood cultures only, 3 (3.3\%) in pleural fluid cultures only, and 1 (1.1\%) each in cerebrospinal fluid and joint fluid cultures only. A further 1 (1.1\%) case had both blood and cerebrospinal fluid cultures positive, 1 (1.1\%) had both blood and joint fluid cultures positive, and 1 (1.1\%) was culture negative, but had a diagnosis of meningitis and a positive streptococcal urinary antigen test, with no other organisms implicated.

When comparing IPD patients $(N=92)$, non-IPD patients $(N=404)$ and non-pneumococcal disease patients $(N=9181)$ (Table 2), significant differences across groups were observed for age (mean age in controls: $72.2 \pm 16.1$ years, non-IPD patients:70.8 \pm 14.7 years, IPD patients: $61.5 \pm 16.3$ years; $p<0.001$ ), gender (with higher proportions of males among IPD and non-IPD patients), history of pneumococcal vaccination (IPD patients: $3.3 \%$, nonIPD patients: $19.1 \%$, controls: $18.5 \%$; $p=0.001$ ), influenza vaccination in the past $1 \mathrm{yr}$ before admission (IPD patients: $6.5 \%$, non-IPD patients: $17.1 \%$, controls: $15.2 \%$; $p=0.039$ ), and Charlson's co-morbidity score (median 
Table 1 Demographic and clinical characteristics and outcomes of hospitalised patients with pneumococcal disease

\begin{tabular}{ll}
\hline Characteristics & $\begin{array}{l}\text { Pneumococcal disease } \\
(\boldsymbol{N}=496), \mathrm{N}(\%)\end{array}$ \\
\hline Age, mean (years) \pm SD & $69.1 \pm 15.4$ \\
Age group & \\
$\quad<65$ years & $170(34.3)$ \\
$65-74$ years & $125(25.2)$ \\
$75-84$ years & $131(26.4)$ \\
$\geq 85$ years & $70(14.1)$ \\
Gender = male & $325(65.5)$ \\
Ethnicity & \\
Chinese & $359(72.4)$ \\
Malay & $61(12.3)$ \\
Indian & $39(7.9)$ \\
Others & $37(7.5)$ \\
Nursing home residence & $4(0.8)$
\end{tabular}

Vaccination status (received $\geq 14$ days prior to admission)

\begin{tabular}{|c|c|}
\hline PCV13 & $6(1.2)$ \\
\hline PPSV23 & $78(15.7)$ \\
\hline Any pneumococcal vaccination & $80(16.1)$ \\
\hline Influenza vaccination in past $1 \mathrm{yr}$ & $75(15.1)$ \\
\hline \multicolumn{2}{|l|}{ Co-morbidities } \\
\hline Charlson's score, median (IQR) & $2(1-4)$ \\
\hline Charlson's score $>3$ & $137(27.6)$ \\
\hline Myocardial infarction/Congestive heart failure & $143(28.8)$ \\
\hline Cerebrovascular disease & $66(13.3)$ \\
\hline Dementia & $54(10.9)$ \\
\hline Chronic pulmonary disease & $149(30.0)$ \\
\hline Liver disease & $27(5.4)$ \\
\hline Diabetes mellitus & $147(29.6)$ \\
\hline Renal disease & $93(18.8)$ \\
\hline Any malignancy & $53(10.7)$ \\
\hline Hemiplegia & $18(3.6)$ \\
\hline Peripheral vascular disease & $22(4.4)$ \\
\hline Peptic ulcer disease & $17(3.4)$ \\
\hline Connective tissue disease & $8(1.6)$ \\
\hline AlDs & $3(0.6)$ \\
\hline \multicolumn{2}{|l|}{ Primary diagnosis } \\
\hline Pneumonia & $347(70.0)$ \\
\hline Sepsis & $63(12.7)$ \\
\hline Meningitis & $5(1.0)$ \\
\hline Other respiratory diseases & $73(14.7)$ \\
\hline Other diagnoses & $8(1.6)$ \\
\hline \multicolumn{2}{|l|}{ Outcomes } \\
\hline ICU admission & $54(11.0)$ \\
\hline Length of stay (days), median (IQR) & $8(5-14)$ \\
\hline
\end{tabular}

Table 1 Demographic and clinical characteristics and outcomes of hospitalised patients with pneumococcal disease (Continued)

\begin{tabular}{ll}
\hline Characteristics & $\begin{array}{l}\text { Pneumococcal disease } \\
(\boldsymbol{N}=496), N(\%)\end{array}$ \\
\hline Length of stay $>7$ days & $275(55.4)$ \\
In-hospital mortality & $70(14.1)$ \\
30-day in-hospital mortality & $33(6.7)$ \\
Readmission for any pneumococcal infection & \\
Within 30 days from discharge date & $1(0.2)$ \\
Anytime during study period & $6(1.2)$
\end{tabular}

$S D$ standard deviation, $P C V 13$ 13-valent pneumococcal conjugate vaccination, PPSV23 23-valent pneumococcal polysaccharide vaccine, IQR inter-quartile range, ICU intensive care unit

score in controls: 3 , non-IPD patients: 2 , IPD patients: 1 ; $p<0.001)$. In terms of clinical outcomes, IPD was associated with the highest proportion of intensive care unit (ICU) admissions $(20.7 \%$, versus $8.7 \%$ in non-IPD patients and $6.3 \%$ in controls; $p<0.001)$ and in-hospital all-cause mortality (26.1\% versus 11.4 and $9.1 \%$ respectively; $\mathrm{p}<0.001)$. Similarly, length of stay was longest for IPD patients (median duration 9 days, versus 8 days in non-IPD and control groups; $p=0.003$ ).

In our multivariable models (Table 2), IPD patients were much less likely than controls to have a history of any pneumococcal vaccination prior to admission $(\mathrm{RRR}=0.20,95 \% \mathrm{CI} 0.06-0.69 ; p=0.011)$. IPD was also positively associated with male gender $(R R R=1.87$, 95\%CI 1.18-2.96; $p=0.008$ ), and negatively associated with increasing age groups, with very elderly patients ( $\geq 85$ years) being less likely to have IPD compared to younger counterparts $(<65$ years $)(p<0.001)$. Although influenza vaccination was negatively associated with IPD on univariate analysis, this factor was non-significant after multivariable adjustment. Non-IPD patients were also more likely than controls to be of male gender, although the effect size was smaller than in IPD patients $(\mathrm{RRR}=1.42,95 \% \mathrm{CI} 1.15-1.76 ; p=0.001)$. Patients $\geq 85$ years and those with Charlson's score of $>3$ were also less likely to have non-IPD, and no significant differences in vaccination status was observed.

Of the 496 patients with pneumococcal disease, 70 (14.1\%) died during their admission (Table 3). The case fatality rates for IPD and non-IPD were 26.1 and $11.4 \%$ respectively. Patients who died were more likely to be older (mean age 74.4 \pm 14.3 years vs $68.2 \pm 15.4$ years; $p<0.001$ ), have a Charlson's score $>3$, a diagnosis of IPD, or ICU admission. Presence of co-morbidities such as myocardial infarction/congestive heart failure, cerebrovascular disease and dementia were also significantly associated with mortality. After adjustments in our multivariable models, factors significantly and independently associated with mortality from pneumococcal disease were age $\geq 85$ years (compared to below 65 years) (adjusted OR, AOR $=10.78$, 
95\%CI 4.10-28.37; $p<0.001$ ), Charlson's score $>3$ (AOR = 1.93, 95\%CI 1.02-3.64; $p=0.042$ ), diagnosis of IPD $(\mathrm{AOR}=3.19,95 \% \mathrm{CI} 1.55-6.59 ; p=0.002)$ and ICU admission $(\mathrm{AOR}=23.22,95 \% \mathrm{CI} 11.07-48.70 ; \mathrm{p}<0.001)$.

\section{Discussion}

Among our cases comprising hospitalised patients with pneumococcal disease, majority were aged $\geq 65$ years and of male gender, and about a quarter had a Charlson's score of $>3$. The ethnic distribution of the patients was reflective of the national census [22]. Compared to the control group, IPD patients demonstrated a trend towards being younger, and with lower Charlson's scores. Non-IPD patients were more similar to the control group, but with only slightly lower mean age and Charlson's scores. Patients in older age groups or with more co-morbidities might have had increased prior healthcare exposures, or had specific risk factors (such as swallowing impairment) which resulted in them being more likely to develop infections [23, 24] from other aetiologies other than S. pneumoniae [25, 26]. However, in contrast to the age and co-morbidity trends, the ICU admission and in-hospital mortality rates were highest in IPD patients and lowest in the control group. This reflects the virulence of the disease and highlights the importance of early recognition, treatment, and prevention where possible.

Our findings showed that prior pneumococcal vaccination was associated with a reduced risk of IPD by about $80 \%$ in our study population, after adjustments for age, gender, Charlson's score and influenza vaccination status. This was consistent with studies in other settings evaluating the effectiveness of PCV13 and PPSV23 [1315]. Pneumococcal serotypes covered by these vaccines have been shown to be well matched to those circulating in the Singapore population $[6,10,27]$. In particular, serotypes $3,6 \mathrm{~B}, 7 \mathrm{~F}, 8,19 \mathrm{~A}$ and $23 \mathrm{~F}$ are predominant in the 19-64 years age group, while serotypes 3, 14 and 19A are predominant among those 65 years and above [27].

In addition, $50(54.4 \%)$ of the IPD cases among our hospital patients were below 65 years of age, of whom 24 (48.0\%) had some form of chronic disease. A Japanese study of 10.4 million individuals demonstrated that younger adults with at least one medical condition were at greater risk of IPD compared to healthy older adults. For example, an adult aged 50-64 years with an underlying medical condition had a higher risk compared to a healthy adult aged $\geq 65$ years [28]. Although the overall incidence in younger adults is lower than in older age groups $[4,29]$, the increased susceptibility of adults $<65$ years with chronic diseases suggests that targeted efforts to vaccinate this population might be beneficial in reducing IPD incidence.
Currently, our hospital has ongoing vaccination programmes to identify and opportunistically vaccinate high-risk groups according to standardised protocols. In the inpatient setting, high-risk inpatients are counselled on and given vaccination prior to discharge, while in the outpatient setting, selected specialist clinics have a nurse-led programme for counselling and vaccination administration for high-risk patients, in conjunction with the medical consultation. Vaccination should also be provided by primary care practitioners as part of chronic disease management and alongside other preventive services such as health screening.

Our study, however, did not demonstrate any significant effect of PCV13 vaccination on reducing risk of pneumococcal disease, likely due to the low proportion of patients in our study with a clearly documented history of PCV13 vaccination. Apart from adult vaccination, widespread introduction of pneumococcal conjugate vaccine in children has been shown to reduce the incidence of IPD across all ages groups due to herd immunity, despite evidence of serotype replacement [30-33]. Singapore introduced PCV7 into its National Childhood Immunisation Programme in October 2009, and this was switched to PCV13 in December 2011. The costeffectiveness of childhood vaccination in the Singapore setting has been demonstrated by Tyo et al. [34]. Future studies are warranted to study the effectiveness of increasing PCV13 vaccination uptake, in both adults and children, to reduce incidence of invasive and noninvasive pneumococcal disease.

Finally, our study also demonstrated key factors associated with in-hospital mortality among pneumococcal patients. ICU admission was the strongest predictor, with those having admission being 23 times as likely as those who did not to die during their admission. Other factors included age $\geq 85$ years, higher Charlson's score and diagnosis of IPD. Medical teams should especially note the increased mortality risk in these patients and ensure prompt management to reduce the risk of death.

The strengths of our study include a systematic selection of cases and controls among hospitalised patients. Furthermore, we followed up the patients longitudinally to the point of discharge from the hospital. Our study population is likely representative of patients hospitalised for pneumococcal disease in Singapore, as our hospital has a large catchment area which is not geographically restricted and patients from anywhere in Singapore may be admitted. Our findings are useful to guide the identification of patients at high risk of in-hospital mortality from the disease. They also support current recommendations to vaccinate at-risk individuals to prevent IPD.

Our study also has some limitations. Selection bias might have been present as clinicians may have preferentially ordered streptococcal urinary antigen and sterile 


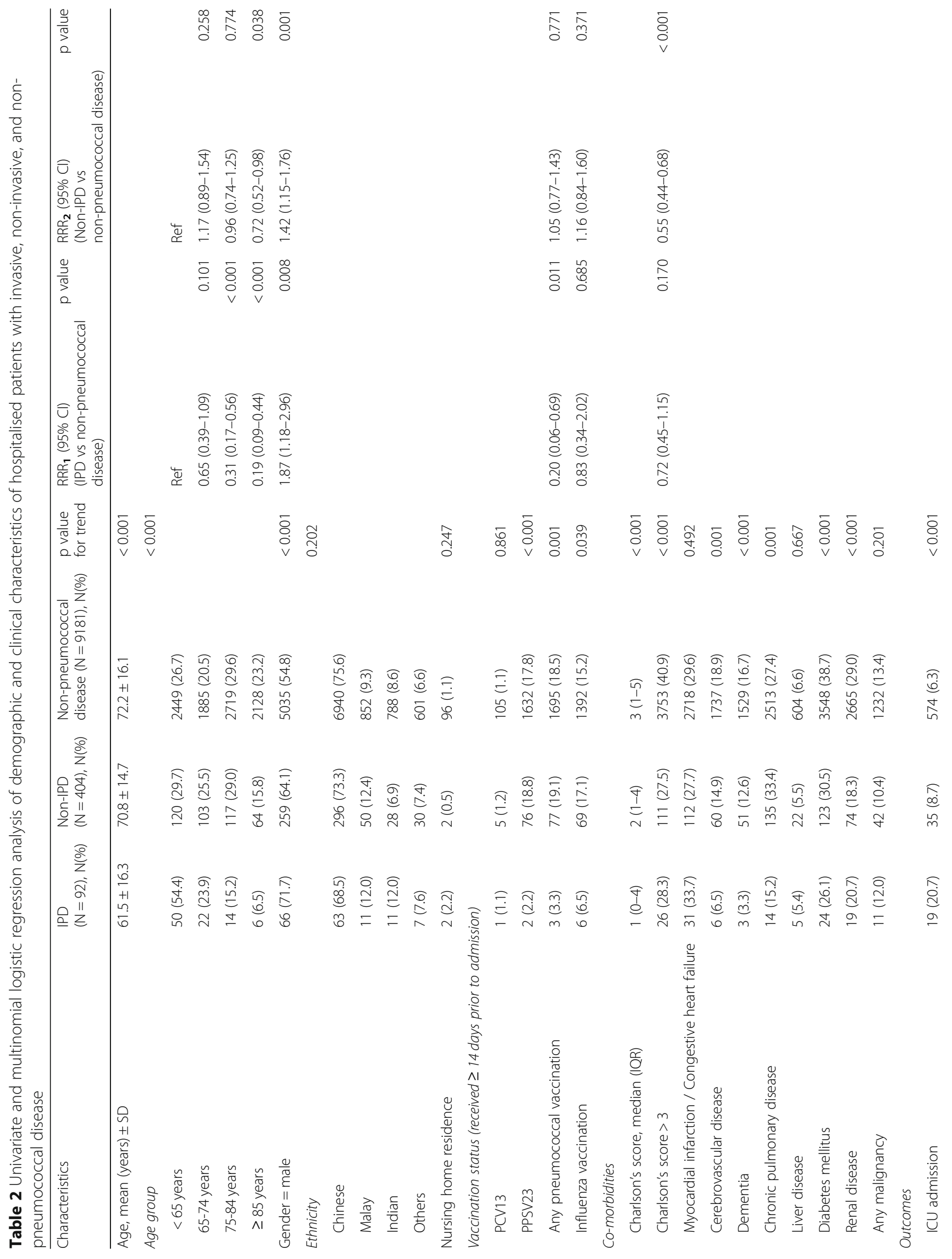




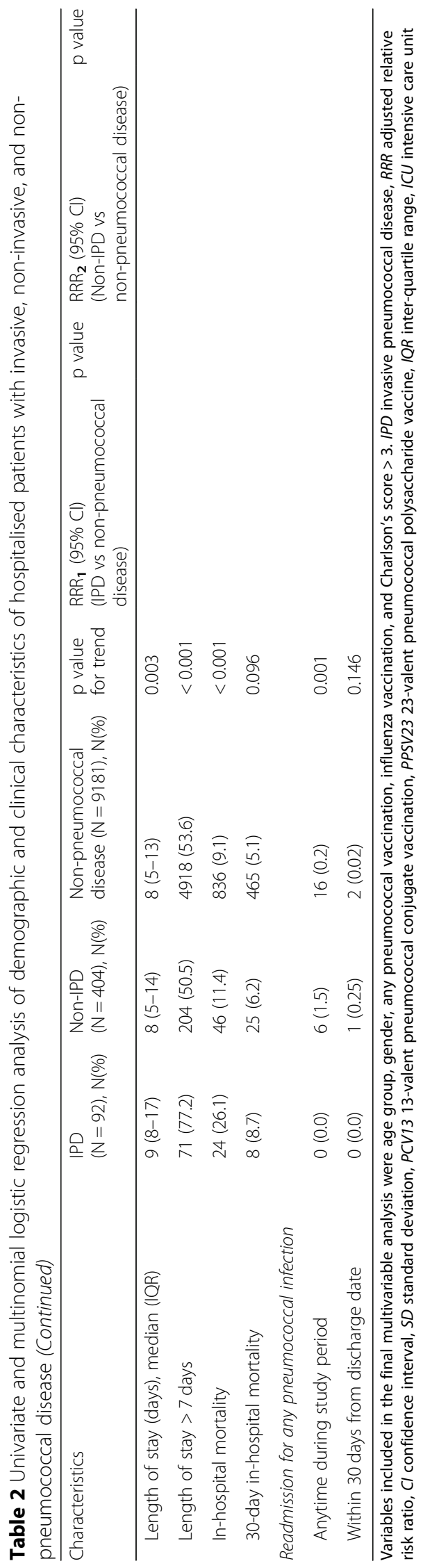


Table 3 Univariate and multiple logistic regression analysis of demographic and clinical factors associated with all-cause mortality among pneumococcal disease patients

\begin{tabular}{|c|c|c|c|c|c|c|}
\hline Characteristics & $\begin{array}{l}\text { Died }(\boldsymbol{N}=70) \\
N(\%)\end{array}$ & $\begin{array}{l}\text { Survived }(\boldsymbol{N}=426) \\
\mathrm{N}(\%)\end{array}$ & OR $(95 \% \mathrm{Cl})$ & $p$ value & AOR $(95 \% \mathrm{Cl})$ & $p$ value \\
\hline Age, mean (years) $\pm S D$ & $74.4 \pm 14.3$ & $68.2 \pm 15.4$ & & $<0.001$ & & \\
\hline \multicolumn{7}{|l|}{ Age group } \\
\hline$<65$ years & $16(22.9)$ & $154(36.2)$ & Ref & Ref & Ref & Ref \\
\hline $65-74$ years & $18(25.7)$ & $107(25.1)$ & $1.62(0.79-3.32)$ & 0.188 & $1.70(0.69-4.15)$ & 0.246 \\
\hline $75-84$ years & $16(22.9)$ & $115(27.0)$ & $1.34(0.64-2.79)$ & 0.435 & $1.99(0.80-4.94)$ & 0.138 \\
\hline$\geq 85$ years & $20(28.6)$ & $50(11.7)$ & $3.85(1.85-8.00)$ & $<0.001$ & $10.78(4.10-28.37)$ & $<0.001$ \\
\hline Gender $=$ male & $45(64.3)$ & $280(65.7)$ & $0.94(0.55-1.59)$ & 0.814 & $1.05(0.55-1.98)$ & 0.892 \\
\hline \multicolumn{7}{|l|}{ Ethnicity } \\
\hline Chinese & $51(72.9)$ & $308(72.3)$ & Ref & Ref & & \\
\hline Malay & $9(12.9)$ & $52(12.2)$ & $1.05(0.49-2.25)$ & 0.910 & & \\
\hline Indian & $7(10.0)$ & $32(7.5)$ & $1.32(0.55-3.15)$ & 0.530 & & \\
\hline Others & $3(4.3)$ & $34(8.0)$ & $0.53(0.16-1.80)$ & 0.311 & & \\
\hline \multicolumn{7}{|c|}{ Vaccination status (received $\geq 14$ days prior to admission) } \\
\hline Any pneumococcal vaccination & $7(10.0)$ & $73(17.1)$ & $0.54(0.24-1.22)$ & 0.138 & & \\
\hline Influenza vaccination in past $1 \mathrm{yr}$ & $8(11.4)$ & $67(15.7)$ & $0.69(0.32-1.51)$ & 0.354 & & \\
\hline \multicolumn{7}{|l|}{ Co-morbidities } \\
\hline Charlson's score, median (IQR) & $1(0-4)$ & $2(1-4)$ & & $<0.001$ & & \\
\hline Charlson's score $>3$ & $30(42.7)$ & $107(25.1)$ & $2.24(1.33-3.77)$ & 0.002 & $1.93(1.02-3.64)$ & 0.042 \\
\hline $\begin{array}{l}\text { Myocardial infarction / Congestive heart } \\
\text { failure }\end{array}$ & $30(42.9)$ & $113(26.5)$ & $2.08(1.24-3.49)$ & 0.006 & & \\
\hline Cerebrovascular disease & $17(24.3)$ & $49(11.5)$ & $2.47(1.33-4.60)$ & 0.004 & & \\
\hline Dementia & $16(22.9)$ & $38(8.9)$ & $3.03(1.58-5.79)$ & 0.001 & & \\
\hline Chronic pulmonary disease & $16(22.9)$ & $133(31.2)$ & $0.65(0.36-1.18)$ & 0.160 & & \\
\hline Liver disease & $3(4.3)$ & $24(5.6)$ & $0.75(0.22-2.56)$ & 0.997 & & \\
\hline Diabetes mellitus & $24(34.3)$ & $123(28.9)$ & $1.29(0.75-2.20)$ & 0.409 & & \\
\hline Renal disease & $16(22.9)$ & $77(18.1)$ & $1.34(0.73-2.47)$ & 0.343 & & \\
\hline Any malignancy & $12(17.1)$ & $41(9.6)$ & $1.94(0.97-3.91)$ & 0.063 & & \\
\hline Invasive pneumococcal disease & $24(34.3)$ & $68(16.0)$ & $2.75(1.57-4.80)$ & $<0.001$ & $3.19(1.55-6.59)$ & 0.002 \\
\hline \multicolumn{7}{|l|}{ Outcomes } \\
\hline ICU admission & $31(44.3)$ & $23(5.4)$ & $\begin{array}{l}13.93(7.40- \\
26.19)\end{array}$ & $<0.001$ & $\begin{array}{l}23.22(11.07- \\
48.70)\end{array}$ & $<0.001$ \\
\hline Length of stay (days), median (IQR) & $7(4-21)$ & $8(6-15)$ & & 0.081 & & \\
\hline Length of stay $>7$ days & $33(47.1)$ & $242(56.8)$ & $1.08(0.90-1.29)$ & 0.414 & & \\
\hline
\end{tabular}

Variables included in the final multivariable analysis were age group, gender, Charlson's score $>3$, diagnosis of invasive pneumococcal disease, and intensive care unit admission. $O R$ odds ratio, $C l$ confidence interval, $A O R$ adjusted $O R, S D$ standard deviation, IQR inter-quartile range, ICU intensive care unit

site cultures for certain groups of patients, such as those who were elderly, with co-morbidities, or without records of recent vaccinations. At the time of the study, we had verified that there were no clinical protocols mandating such tests to be done for any particular patient groups. Our definition of cases included the use of results from streptococcal urinary antigen testing, which has an estimated sensitivity of $74.0 \%$ and specificity of 97.2\% [35]. Sterile site cultures from multiple possible sources (e.g. blood, joint fluid, or pleural fluid) will also have varying microbial culture methods with different sensitivity and specificity. Some cases of pneumococcal disease may hence have been misclassified as controls (i.e. false negatives). However, such a misclassification could have reduced the magnitude of our findings, but not negated them. As our data was obtained through hospital electronic medical records, clinical data from other sources (e.g. other hospitals or the primary care sector) were not available. However, majority of our patients tended to return back to our hospital if future 
admissions were required. Moreover, it is likely that this issue would result in non-differential misclassification (if any) as data would not be captured differently across study groups. We were unable to capture data for some known risk factors, such as smoking history and socioeconomic status. Our analysis of IPD cases was limited by the relatively small numbers, especially when divided into subgroups. Pneumococcal serotype data were also not available for this study, as this is not routinely performed for isolates. Nevertheless, we have reviewed data from serotyping studies conducted in the local setting $[6,10,27]$, which we believe to be applicable to our study population. The generalisability of our study is limited to hospitalised adult patients.

\section{Conclusion}

In conclusion, while patients with pneumococcal disease tended to be younger and with less co-morbidities compared to those without pneumococcal disease, risk of ICU admission and death were nevertheless higher, especially in IPD patients. Mortality risk was highest in those with ICU admission, age $\geq 85$ years, higher Charlson's score and diagnosis of IPD. Pneumococcal vaccination effectiveness against IPD was estimated to be about $80 \%$, and should be encouraged among high-risk patients, including those from younger age groups.

\section{Abbreviations}

IPD: Invasive pneumococcal disease; ICU: Intensive care unit;

CAP: Community-acquired pneumonia; PCV13: 13-valent pneumococcal conjugate vaccine; PPSV23: 23-valent pneumococcal polysaccharide vaccine; ACIP: Advisory Committee on Immunisation Practices; $\mathrm{MOH}$ : Ministry of Health; TTSH: Tan Tock Seng Hospital; ICD-10: International Classification of Diseases, 10th Revision; IQR: Interquartile range; RRR: Relative risk ratio; OR: Odds ratio; Cl: Confidence interval; AOR: Adjusted odds ratio

\section{Acknowledgments}

Not applicable.

\section{Authors' contributions}

TC and MZT analysed and interpreted the data collected for this study, and contributed to the writing of the manuscript. WMK and AC contributed to study design, interpretation of data, and critically reviewed the manuscript. $\mathrm{HJH}$ conceived the study, analysed and interpreted the data, and was a major contributor in writing the manuscript. All authors approved the final version of the manuscript for submission.

\section{Funding}

Not applicable.

\section{Availability of data and materials}

The datasets used and/or analysed during the current study are available from the corresponding author on reasonable request.

\section{Ethics approval and consent to participate}

Ethics approval for this study was obtained from the Domain-Specific Review Board of the National Healthcare Group (NHG DSRB No: 2017/00347). This was a retrospective study using anonymised data and informed consent was not required.

\section{Consent for publication}

Not applicable.

\section{Competing interests}

The authors declare that they have no competing interests.

\section{Author details}

'Department of Clinical Epidemiology, Office of Clinical Epidemiology, Analytics, and Knowledge, Tan Tock Seng Hospital, 11 Jalan Tan Tock Seng, Singapore 308433, Singapore. ${ }^{2}$ Preventive Medicine Residency Programme, National University Health System, Singapore, Singapore.

Received: 3 August 2019 Accepted: 8 June 2020

Published online: 17 June 2020

\section{References}

1. Said MA, Johnson HL, Nonyane BA, Deloria-Knoll M, O'Brien KL. Estimating the burden of pneumococcal pneumonia among adults: a systematic review and meta-analysis of diagnostic techniques. PLoS One. 2013;8(4): e60273.

2. Song JH, Oh WS, Kang Cl, Chung DR, Peck KR, Ko KS, et al. Epidemiology and clinical outcomes of community-acquired pneumonia in adult patients in Asian countries: a prospective study by the Asian network for surveillance of resistant pathogens. Int J Antimicrob Ag. 2008;31:107-14.

3. Drijkoningen JJC, Rohde GGU. Pneumococcal infection in adults: burden of disease. Clin Microbio Infect. 2014;20(Suppl. 5):45-51.

4. Low S, Chan FLF, Cutter J, Ma S, Goh KT, Chew SK. A national study of the epidemiology of pneumococcal disease among hospitalised patients in Singapore: 1995-2004. Singap Med J. 2007:48(9):824-9.

5. Jackson ML, Neuzil KM, Thompson WW, Shay DK, Yu O, Hanson CA, et al. The burden of community-acquired pneumonia in seniors: results of a population-based study. Clin Infect Dis. 2004;39(11):1642-50.

6. Hsu LY, Lui SW, Lee JL, Hedzlyn HM, Kong DH, Shameen S, et al. Adult invasive pneumococcal disease pre- and peri- pneumococcal conjugate vaccine introduction in a tertiary hospital in Singapore. J Med Microbiol. 2009:58(Pt 1):101-4.

7. Bogaert D, de Groot R, Hermans PWM. Streptococcus pneumoniae colonisation: the key to pneumococcal disease. Lancet Infect Dis. 2004;4: 144-54.

8. Lynch JP 3rd, Zhanel GG. Streptococcus pneumoniae: epidemiology, risk factors, and strategies for prevention. Semin Respir Crit Care Med. 2009; 30(2):189-209.

9. File TM Jr. Streptococcus pneumoniae and community-acquired pneumonia: a cause for concern. Am J Med. 2004;117(Suppl 3A):39S-50S.

10. Vasoo S, Singh K, Chow C, Tzer RPL, Hsu LY, Tambyah PA. Pneumococcal carriage and resistance in children attending day care centres in Singapore in an early era of PCV-7 uptake. J Inf Secur. 2010;60(June 6):507-9.

11. Pletz MW, Rohde GG, Welte T, Kolditz M, Ott S. Advances in the prevention, management, and treatment of community-acquired pneumonia. F1000Res. 2016;5:F1000 Faculty Rev-1300.

12. Eng P, Lim LH, Loo CM, Low JA, Tan C, Tan EK, et al. Role of pneumococcal vaccination in prevention of pneumococcal disease among adults in Singapore. Int J Gen Med. 2014;7:179-91.

13. Falkenhorst $G$, Remschmidt $C$, Harder $T$, Hummers-Pradier E, Wichmann $\mathrm{O}$, Bogdan C. Effectiveness of the 23-valent pneumococcal polysaccharide vaccine (PPV23) against pneumococcal disease in the elderly: systematic review and meta-analysis. PloS One. 2017;12(1):e0169368.

14. Bonten MJ, Huijts SM, Bolkenbaas M, Webber C. Polysaccharide conjugate vaccine against pneumococcal pneumonia in adults. N Engl J Med. 2015; 372(12):1114-25.

15. Golos M, Eliakim-Raz N, Stern A, Leibovici L, Paul M. Conjugated pneumococcal vaccine versus polysaccharide pneumo-coccal vaccine for prevention of pneumonia and invasive pneumococcal disease in immunocompetent and immunocompromised adultsand children. Cochrane Database Syst Rev. 2016;8:CD012306.

16. Tomczyk S, Bennett NM, Stoecker C, Gierke R, Moore MR, Whitney CG, et al. Use of 13-valent pneumococcal conjugate vaccine and 23-valent pneumococcal polysaccharide vaccine among adults aged $\geq 65$ years: recommendations of the advisory committee on immunization practices (ACIP). MMWR Morb Mortal Wkly Rep. 2014;63(37):822-5.

17. Ministry of Health, Singapore. National Immunisation Schedule 2017. https:// www.moh.gov.sg/content/moh_web/home/pressRoom/ pressRoomltemRelease/2017/moh-establishes-national-adult-immunisationschedule\%2D\%2Dextends-us.html. Accessed 5 Jun 2020. 
18. Ho HJ, Chan YY, Ibrahim MAB, Wagie AA, Wong CM, Chow A. A formative research-guided educational intervention to improve the knowledge and attitudes of seniors towards influenza and pneumococcal vaccinations. Vaccine. 2017:35(47):6367-74.

19. Bravo LC. Asian strategic Alliance for pneumococcal disease prevention (ASAP) working group. Overview of the disease burden of invasive pneumococcal disease in Asia. Vaccine. 2009;27(52):7282-91.

20. Hung IF, Tantawichien T, Tsai YH, Patil S, Zotomayor R. Regional epidemiology of invasive pneumococcal disease in Asian adults: epidemiology, disease burden, serotype distribution, and antimicrobial resistance patterns and prevention. Int J Infect Dis. 2013;17(6):e364-73.

21. Quan H, Sundararajan V, Halfon P, Fong A, Burnand B, Jean-Christophe L, et al. Coding algorithms for defining comorbidities in ICM-9-CM and ICD-10 administrative data. Med Care. 43(11):1130-9.

22. Department of Statistics, Singapore. Population Trends, 2017. https://www. singstat.gov.sg/-/media/files/publications/population/population2017.pdf. Accessed 5 Jun 2020.

23. Fung $\mathrm{HB}$, Monteagudo-Chu MO. Community-acquired pneumonia in the elderly. Am J Geriatr Pharmac. 2010;8(1):47-62.

24. Weinberger B, Herndler-Brandstetter D, Schwanninger A, Weiskopf D, Grubeck-Loebenstein B. Biology of immune responses to vaccines in elderly persons. Vaccine. 2008:46:1078-84.

25. Gavazzi G, Krause K-H. Ageing and infection. Lancet Infect Dis. 2002;2(11): 659-66.

26. Yoshikawa $\Pi$ T. Epidemiology and unique aspects of aging and infectious diseases. Clin Infect Dis. 2000;30(6):931-3.

27. Jauneikaite E, Jefferies JMC, Churton NWV, Tzer RPL, Hibberd ML, Clarke SC. Genetic diversity of Streptococcus pneumonia causing meningitis and sepsis in Singapore during the first year of PCV7 implementation. Emerg Microbes Infect. 2014;3:e39

28. Imai K, Petigara T, Kohn MA, Nakashima K, Aoshima M, Shito A, et al. Risk of pneumococcal diseases in adults with underlying medical conditions: a retrospective, cohort study using two Japanese healthcare databases. BMJ Open. 2018;8(3):e018553.

29. Marrie TJ, Tyrrell GJ, Sumit RM, Eurcih DT. Effect of age on the manifestations and outcomes of invasive pneumococcal disease in adults. Am J Med. 2018;131(1):100.e101-7.

30. Flasche S, Van Hoek AJ, Goldblatt D, Edmunds WJ, O'Brien KL, Scott JA, et al. The potential for reducing the number of pneumococcal conjugate vaccine doses while sustaining herd immunity in high-income countries. PLoS Med. 2015;12(6):e1001839.

31. McIntosh ED, Conway P, Willingham J, Hollingsworth R, Llyod A. Pneumococcal pneumonia in the UK--how herd immunity affects the costeffectiveness of 7-valent pneumococcal conjugate vaccine (PCV). Vaccine. 2005;23(14):1739-45.

32. Pletz MW, Maus U, Hohlfeld JM, Lode H, Welte T. Pneumococcal vaccination: conjugated vaccine induces herd immunity and reduces antibiotic resistance. Dtsch Med Wochenschr. 2008;133(8):358-62.

33. Stephens DS. Protecting the herd: the remarkable effectiveness of the bacterial meningitis polysaccharide-protein conjugate vaccines in altering transmission dynamics. Trans Am Clin Climatol Assoc. 2011;122:115-23.

34. Tyo KR, Rosen MM, Zeng W. Cost-effectiveness of conjugate pneumococcal vaccination in Singapore: comparing estimates for 7-valent, 10-valent, and 13-valent vaccines. Vaccine. 2011;29(38):6686-94.

35. Sinclair A, Xie X, Teltscher M, Dendukuri N. Systematic review and metaanalysis of a urine-based pneumococcal antigen test for diagnosis of community-acquired pneumonia caused by Streptococcus pneumoniae. J Clin Microbiol. 2013;51(7):2303-10.

\section{Publisher's Note}

Springer Nature remains neutral with regard to jurisdictional claims in published maps and institutional affiliations.

Ready to submit your research? Choose BMC and benefit from:

- fast, convenient online submission

- thorough peer review by experienced researchers in your field

- rapid publication on acceptance

- support for research data, including large and complex data types

- gold Open Access which fosters wider collaboration and increased citations

- maximum visibility for your research: over $100 \mathrm{M}$ website views per year

At BMC, research is always in progress.

Learn more biomedcentral.com/submissions 